ARTICLE

\title{
Direct measurement of individual phonon lifetimes in the clathrate compound $\mathrm{Ba}_{7.81} \mathrm{Ge}_{40.67} \mathrm{Au}_{5.33}$
}

Pierre-François Lory (10) 1,2, Stéphane Pailhès ${ }^{3}$, Valentina M. Giordano ${ }^{3}$, Holger Euchner (10 4, Hong Duong Nguyen ${ }^{5}$, Reiner Ramlau ${ }^{5}$, Horst Borrmann ${ }^{5}$, Marcus Schmidt ${ }^{5}$, Michael Baitinger ${ }^{5}$, Matthias Ikeda 6 , Petr Tomeš ${ }^{6}$, Marek Mihalkovič ${ }^{7}$, Céline Allio ${ }^{8}$, Mark Robert Johnson ${ }^{1}$, Helmut Schober ${ }^{1,9}$, Yvan Sidis ${ }^{10}$, Frédéric Bourdarot ${ }^{11}$, Louis Pierre Regnault ${ }^{11}$, Jacques Ollivier ${ }^{1}$, Silke Paschen ${ }^{6}$, Yuri Grin ${ }^{5} \&$ Marc de Boissieu ${ }^{2}$

Engineering lattice thermal conductivity requires to control the heat carried by atomic vibration waves, the phonons. The key parameter for quantifying it is the phonon lifetime, limiting the travelling distance, whose determination is however at the limits of instrumental capabilities. Here, we show the achievement of a direct quantitative measurement of phonon lifetimes in a single crystal of the clathrate $\mathrm{Ba}_{7.81} \mathrm{Ge}_{40.67} \mathrm{Au}_{5.33}$, renowned for its puzzling 'glass-like' thermal conductivity. Surprisingly, thermal transport is dominated by acoustic phonons with long lifetimes, travelling over distances of 10 to $100 \mathrm{~nm}$ as their wave-vector goes from 0.3 to $0.1 \AA^{-1}$. Considering only low-energy acoustic phonons, and their observed lifetime, leads to a calculated thermal conductivity very close to the experimental one. Our results challenge the current picture of thermal transport in clathrates, underlining the inability of state-of-the-art simulations to reproduce the experimental data, thus representing a crucial experimental input for theoretical developments.

\footnotetext{
${ }^{1}$ Institut Laue-Langevin, Grenoble F-38000, France. ${ }^{2}$ University Grenoble Alpes, CNRS, Grenoble-INP, SIMaP, F-38000 Grenoble, France. ${ }^{3}$ University Lyon, University Claude Bernard Lyon 1, CNRS, Institute of Light and Matter, F-69622 Villeurbanne, France. ${ }^{4}$ Institute of Materials Science and Technology, Vienna University of Technology, 1040 Vienna, Austria. ${ }^{5}$ Max-Planck-Institut für Chemische Physik fester Stoffe, 01187 Dresden, Germany. ${ }^{6}$ Institute of Solid State Physics, Vienna University of Technology, 1040 Vienna, Austria. ${ }^{7}$ Institute of Physics, Slovak Academy of Sciences, 84511 Bratislava, Slovakia.

${ }^{8}$ Physikalisches Institut, Goethe-University, 60438 Frankfurt, Germany. ${ }^{9}$ University Grenoble Alpes, UFR PhITEM, F-38000 Grenoble, France. ${ }^{10}$ Laboratoire Léon Brillouin, CNRS, CEA, UMR-12, 91191 Gif sur Yvette, France. ${ }^{11}$ University Grenoble Alpes, CEA, INAC, F-38000 Grenoble, France. Correspondence and requests for materials should be addressed to S.P. (email: stephane.pailhes@univ-lyon1.fr) or to M.B.(email: Marc.de-Boissieu@simap.grenoble-inp.fr)
} 
A central issue in the development of modern nano- and microtechnology is the engineering of thermal conductivity of semiconducting materials. Thermal energy here is mainly carried by atomic vibrations, described by quasiparticles called phonons. During its lifetime, a phonon transports a quantum of heat, given by the product of its energy and its velocity. The knowledge of individual phonon properties is thus fundamental for applications such as waste heat recovery through thermoelectric (TE) conversion, where one of the main challenges is to identify semiconducting materials with low thermal conductivity ${ }^{1-3}$. As a rule of thumb, low thermal conductivity can be achieved by lowering phonon lifetimes, a strategy which has led to an intensive research activity in the 'phonon engineering' of TE materials. While phonon energies and velocities can be relatively easily measured and calculated, to access phonon lifetimes is extremely challenging both experimentally, because of the limited instrumental resolution of state of the art experimental techniques, and theoretically, as it requires the determination of anharmonicity and a detailed modeling of disorder. Recently, considerable progress has been made in ab initio computational methods, mainly on semiconductors with relatively simple structures $^{4-12}$. In these systems the lifetime reduction due to three-phonon Umklapp processes has been calculated and successfully compared to experimental values of the thermal conductivity. As an example, phonons mostly contributing to the thermal conductivity were reported to have at room temperature mean free paths (MFP) in the $7-70 \mathrm{~nm}$ range in $\mathrm{Bi}_{2} \mathrm{Te}_{3}$ (5 atoms per unit cell) and $2-10 \mathrm{~nm}$ in $\mathrm{PbTe}$, with a $1 / T$ temperature dependence in the high temperature limit. The effect of mass disorder has been calculated only in the case of simple element alloys ${ }^{11,13-15}$, such as $\mathrm{Si}_{0.5} \mathrm{Ge}_{0.5}$, where the relevant mean free path was found to be $0.1-5 \mu \mathrm{m}$.

Face to these recent computational advances, it is necessary to validate the theoretical predictions by actual measurements of the lifetimes of individual phonon states. The phonon lifetime is related to the intrinsic inelastic scattering signal width (full width at half maximum FWHM) by the relation $\tau^{-1}=\pi$. $\Gamma_{\text {FWHM }}$, where $\Gamma_{\text {FWHM }}$ is the signal FWHM expressed in $\mathrm{THz}^{16}$. A typical inelastic neutron scattering (INS) experiment allows measuring lifetimes up to $\tau \sim 5 \mathrm{ps}$ (corresponding to a phonon energy full-width at half maximum $\Gamma_{\text {FWHM }} \sim 250 \mu \mathrm{eV}$ ), which give a MFP of the order of $10 \mathrm{~nm}$ for a phonon group velocity of $2000 \mathrm{~m} \cdot \mathrm{s}^{-1}$. As a result, effective phonon lifetimes have been accurately measured only in a few cases where they are already very short, such as in $\mathrm{PbTe}\left(\Gamma_{\mathrm{FWHM}} \sim 400 \mu \mathrm{eV}, \tau \sim 3\right.$ ps for $E \sim 1.5 \mathrm{meV})^{17-19}$ and $\mathrm{Na}_{0.8} \mathrm{Co}_{2}\left(\Gamma_{\mathrm{FWHM}} \sim 625 \mu \mathrm{eV}, \tau \sim 2 \mathrm{ps}\right)^{20}$ or in nanostructured materials with nanometric grains such as AgSbTe $\left(\Gamma_{\text {FWHM }} \sim 500 \mu \mathrm{eV}, \tau \sim 2.6 \text { ps for } E \sim 1.5 \mathrm{meV}\right)^{19,21}$. The neutron resonant spin-echo technique (NRSE) allows for accessing much longer lifetimes, but up to now it has been applied only to simple systems such as $\mathrm{Pb}$ and $\mathrm{Nb}$, where lifetimes in the range 25-90 ps $\left(\Gamma_{\mathrm{FWHM}}=15-5 \mu \mathrm{eV}\right)$ have been reported ${ }^{22}$.

Low thermal conductivities have also been observed in structurally complex crystals with a large number of atoms in the unit cell (>50 atoms), such as guest-host structures (clathrates and skutterudites), where however phonon lifetimes result beyond the standard resolution limit. These materials, known as cage
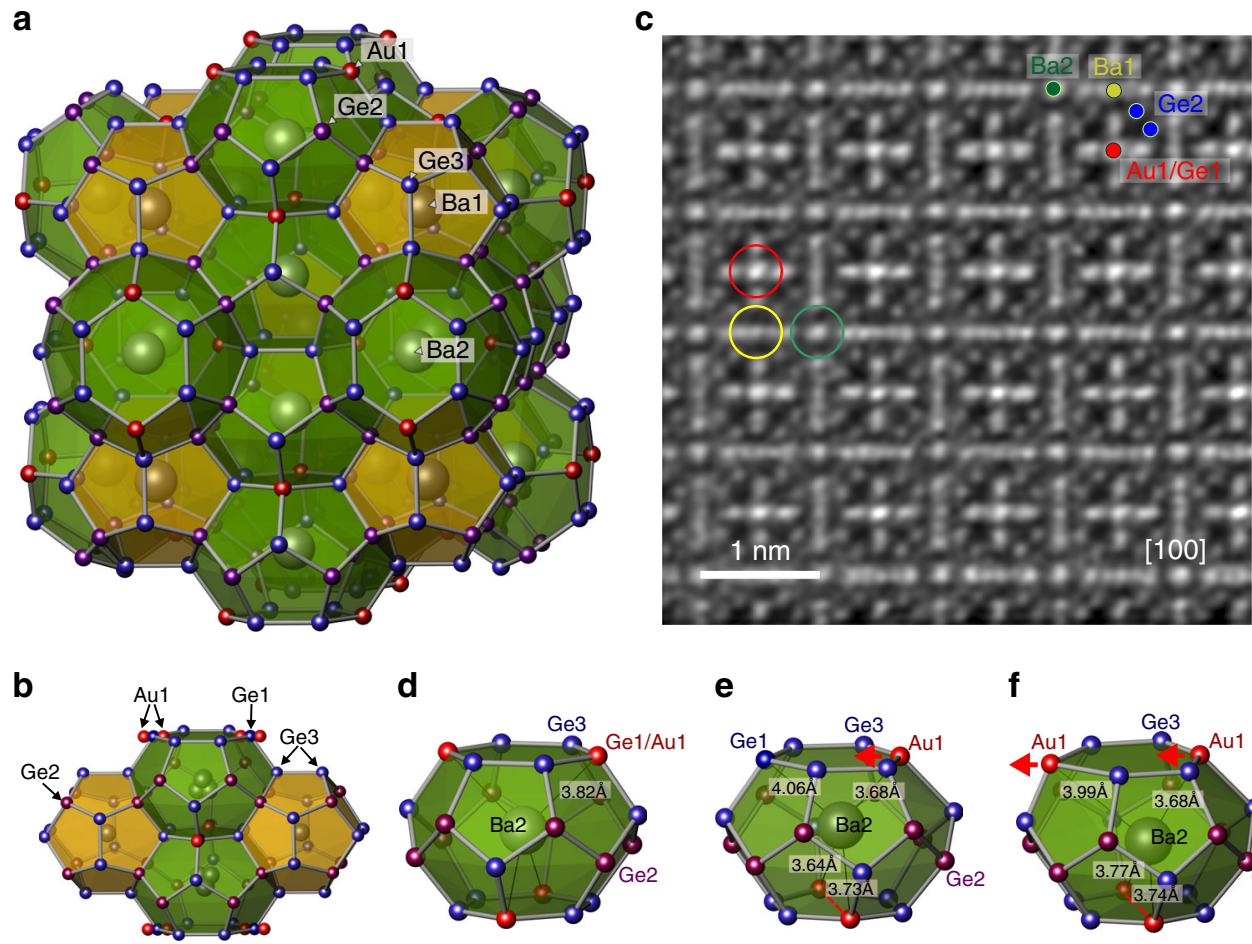

d

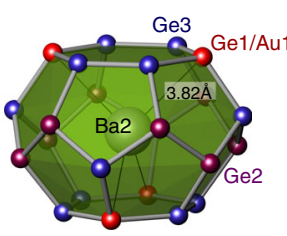

e

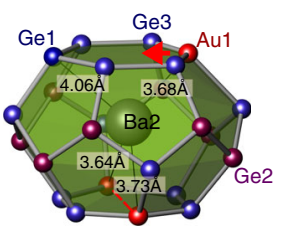

f

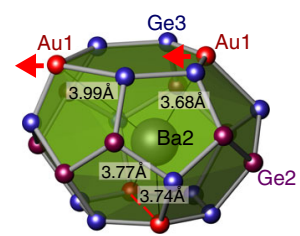

Fig. 1 Crystal structure and local atomic arrangements of $\mathrm{Ba}_{7.81} \mathrm{Ge}_{40.67} \mathrm{Au}_{5.33}$. a Ordered clathrate-I model with the composition $\mathrm{Ba}{ }_{8} \mathrm{Ge}_{40} \mathrm{Au}_{6}$ showing the three-dimensional $\mathrm{Ge} / \mathrm{Au}$ framework with 20-vertices polyhedra (orange) and 24-vertices polyhedra (green), occupied by Ba1 (Wyckoff site 2a) and Ba2 (6d) atoms, respectively. Au atoms on the $6 c$ site are shown in red. b Atomic arrangements around Ba2 and Ge1/Au1 positions; red arrows mark the shift of the Au1 atoms with respect to the $6 c$ site. c Atomic-resolution Scanning Transmission Electron Spectroscopy (STEM) image along the [100] direction reveals strong displacement of atoms from the ideal positions in the regions of Ba2 (green) and Au1/Ge1 (red) positions in comparison with the Ba1 ones (yellow). Additional deviations from the translational symmetry can be recognized in several regions of the image. $\mathbf{d}$ Ideal ordered arrangement in the Ba2 polyhedron with $\mathrm{Ba} 2$ in $6 d$ site and equivalent Ba2-Ge1-Au1 distances for a hypothetical undistorted model. e $\mathrm{A}$ possible atomic arrangement in the Ba2 polyhedron with three Au atoms at Au1 site, Ba2 displaced from the $6 d$ site and smaller Ba2-Au1 distances due to the Ba-Au interactions; $\mathbf{f}$ Atomic arrangement with $3 \mathrm{Au}$ atoms as obtained by using positions from ab initio calculation on a $2 \times 2 \times 2$ supercell and the experimental lattice parameter. The distances compare well with experimental data in $\mathbf{e}$ 

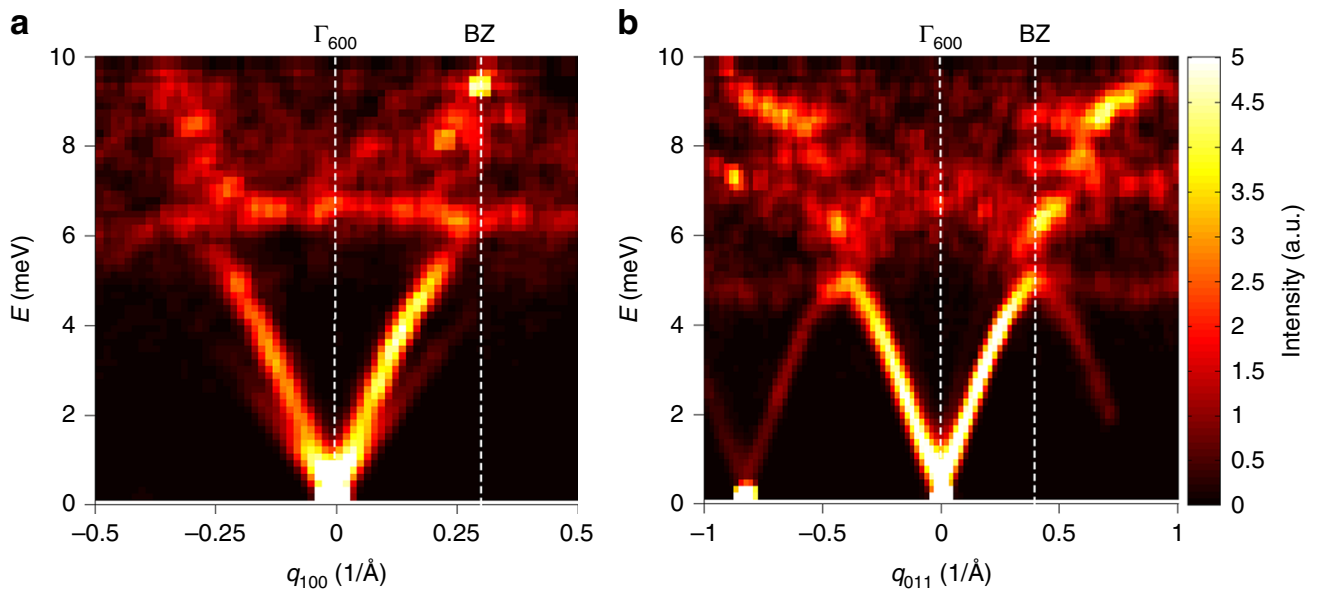

Fig. 2 Experimental surface mapping of the longitudinal acoustic (LA) and transverse acoustic (TA) phonon dispersions in $\mathrm{Ba}_{7.81} \mathrm{Ge}_{40.67} \mathrm{Au}_{5.33} \mathrm{measured} \mathrm{at}$ $300 \mathrm{~K}$. Two-dimensional inelastic neutron scattering intensity distribution as measured on the neutron time-of-flight spectrometer (IN5@ILL) at room temperature around the strong zone center $\Gamma_{600}\left(Q=3.6 \AA^{-1}\right)$. a LA phonons measured along the [100] direction. Well-defined propagating excitations are only observed for LA modes with an energy lower than $6 \mathrm{meV}$. b TA phonons propagating along [011] and polarized along [100]. Well-defined propagating excitation are only observed for TA modes with an energy lower than $5 \mathrm{meV}$

compounds, are composed of covalently bonded polyhedra filled by guest atoms (see Fig. 1a for clathrates) and are characterized by a low thermal conductivity of typically $\kappa$ about $1-2 \mathrm{~W} \mathrm{~m}^{-1} \mathrm{~K}^{-1}$ at room temperature ${ }^{23}$, which is assumed to be related to the presence of lowenergy, non-dispersive optical phonons ${ }^{24-29}$. Formerly associated with isolated vibrations of the guest atoms ${ }^{26,30}$, more recent investigations indicate that these optic modes hybridize with the host matrix, leading to an energy cutoff for the propagative acoustic phonons, and their localisation above $\mathrm{it}^{27,29}$. Moreover, in some clathrates the typical Umklapp peak in the $\kappa(T)$ dependence is smoothed out and replaced by a weakly temperature dependent 'plateau' ${ }^{23}, 31$, that has frequently been referred to as 'glass-like'. In glasses, this is due to very short phonon mean free paths, which have been supposed to exist also in clathrates, possibly caused by twophonon processes like resonant scattering on guest atoms ${ }^{32}, 33-37$, or three-phonon processes due to anharmonicity. Indeed, ab initio calculations in clathrates and other complex material ${ }^{37-40}$ indicate that the many low-lying optic modes enhance the occurrence of three-phonon processes, reducing the acoustic phonons lifetime. However, to date, no experimental evidence of a marked reduction in phonon lifetimes has been found via inelastic neutron/X-ray scattering techniques, mainly because of the limited energy resolution achieved in those experiments ${ }^{24,26,27,29}$.

Here, we tackle this problem, by successfully achieving the hard task of measuring the longest ever reported lifetimes for acoustic phonons in a large single crystal of the clathrate $\mathrm{Ba}_{7.81} \mathrm{Ge}_{40.67} \mathrm{Au}_{5.33}$ (about 54 atoms in the unit cell) whose measured lattice thermal conductivity at $300 \mathrm{~K}$ is of $1.1 \mathrm{~W} \mathrm{~m}^{-1} \mathrm{~K}^{-1}$ (see Methods and Supplementary Note 3 ). This result has been possible thanks to the remarkable crystalline quality (bulk mosaicity of $0.01^{\circ}$, see Methods and Supplementary Note 1) achieved, allowing for unprecedented high-resolution measurements of the acoustic phonon lifetime by means of INS and NRSE. These measurements are supported by ab initio density functional theory (DFT) simulations, and allow us to experimentally confirmed of the long-established thermal transport modeling in complex systems.

\section{Results}

Structural characterization of $\mathrm{Ba}_{7.81} \mathrm{Ge}_{\mathbf{4 0 . 6 7}} \mathbf{A u}_{5.33}$. The importance of achieving a detailed understanding of the atomic structure, including disorder, has been recently highlighted for $\mathrm{AgSbTe}^{21}$, where deviations from the ideal, periodic model significantly affect thermal properties.
$\mathrm{Ba}_{8} \mathrm{Ge}_{46-x} \mathrm{Au}_{x}$ is a clathrate of type I (space group $P m \overline{3} n$ ), made of two kinds of face-sharing Ba-filled cages: a dodecahedral one (20 vertices) and a larger tetrakaidecahedral one (24 vertices; Fig. 1a). At the hypothetical composition $\mathrm{Ba}_{8} \mathrm{Ge}_{40} \mathrm{Au}_{6}$ and in a fully ordered model, 4 gold atoms substitute $\mathrm{Ge}$ in this latter cage ( $6 c$ site), in a distorted tetrahedral arrangement around the central Ba atom (Fig. 1b), however significant deviations from this ideal ordered model have been previously reported ${ }^{41}$. We have here performed a detailed X-ray diffraction investigation, which confirms these findings, revealing that the composition of the unit cell is $\mathrm{Ba}_{7.81} \mathrm{Ge}_{40.67} \mathrm{Au}_{5.33}$ (Supplementary Note 2) and shows that the $\mathrm{Ba} 2$ atoms in the large cages are off-centred and do not occupy the high symmetry position $(6 d)$ any more. They are at an average distance of $0.26 \AA$ from the ideal one, the site being occupied at $96.8 \%$, with a typical inter-defect (vacancy) distance of about $4 \mathrm{~nm}$. Moreover, as a consequence of the covalent $\mathrm{Ba}-\mathrm{Au}$ bonding ${ }^{41}, \mathrm{Au}$ atoms substitute $\mathrm{Ge}$ atoms only partially (about $88.4 \%)$ and are off-centred by $0.08 \AA$ out of the high symmetry (6c) position, the corresponding characteristic inter-defect distance being of approximatively $1.5 \mathrm{~nm}$.

High-end transmission-electron microscopy and STEM images on the same crystal (see Methods) confirmed clearly those results, with $\mathrm{Ba} 2$ and $\mathrm{Au} / \mathrm{Ge} 1$ atoms displaced from their ideal positions, resulting in deviations from the translational symmetry with a wide spectrum of characteristic distances as seen Fig. 1c.

The disorder has been studied by ab initio DFT simulations at $0 \mathrm{~K}$ and by DFT molecular dynamics at $300 \mathrm{~K}$ (Supplementary Note 2). A stable atomic arrangement was obtained using a $2 \times 2 \times 2$ supercell model with chemical composition close to the experimental one and randomly distributed $\mathrm{Ge}$ and $\mathrm{Au}$ atoms. About half of the $\mathrm{Ba} 2$ atoms in the large cages are found to be surrounded only by three gold atoms, with the Ba2 shifting by $0.27 \AA$ towards the Au-triangle, in good agreement with the experimentally determined structure (Fig. 1d-f). A good agreement was also obtained between the experimental structure and the time averaged structure simulated by DFT molecular dynamics.

Measurements of the phonon spectra in $\mathrm{Ba}_{7.81} \mathrm{Ge}_{40.67} \mathrm{Au}_{5.33}$. Lattice dynamics investigations were carried out by INS, on the same single crystal $\mathrm{Ba}_{7.81} \mathrm{Ge}_{40.67} \mathrm{Au}_{5.33}$, and by ab initio calculations using an ideally ordered cubic model, $\mathrm{Ba}_{8} \mathrm{Ge}_{40} \mathrm{Au}_{6}$ 

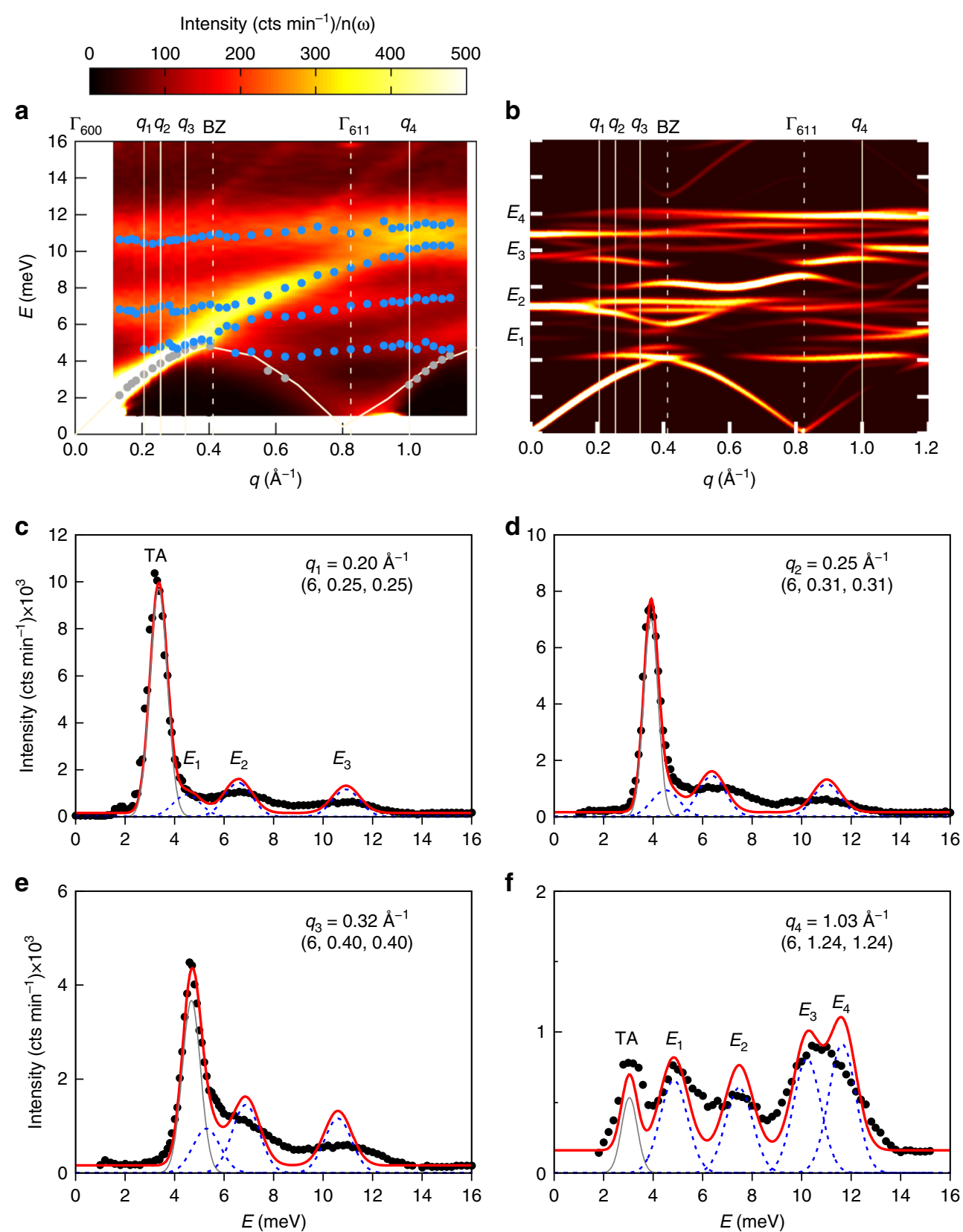

Fig. 3 Transverse acoustic (TA) phonon spectra in $\mathrm{Ba}_{7.81} \mathrm{Ge}_{40.67} \mathrm{Au}_{5.33}$ : experiment vs. simulation at $300 \mathrm{~K}$. a Two dimensional neutron intensity distribution as experimentally measured on a neutron triple axis spectrometer (2T@LLB), along the reciprocal direction ( $6, h, h)$ from the Brillouin zone centre $\Gamma_{600}\left(Q=3.6 \AA^{-1}\right)$ showing the $\mathrm{TA}_{011}^{100}$ dispersion and its hybridization with low-lying optic $\mathrm{Ba}$ and Au modes (red is higher intensity). Grey and blue full circles stand for acoustic and optical excitations respectively as extracted from individual constant q-scans. The solid white line is the $\mathrm{TA}_{011}^{100}$ dispersion relation fitted from the individual constant q-scans as reported in $\mathbf{c - f}$ ). $\mathbf{b}$ Two-dimensional neutron intensity distribution obtained by harmonic simulation in the density functional theory. A different scaling factor has been applied for the acoustic (1.4) and optical (1.2) contributions (see text). Experimental points are shown as grey (acoustic) and blue (optic) full circles, respectively. c-f example of four constant $q$-scans, $q_{1}$ to $q_{4}$ as indicated by the white vertical lines in the mapping $(\mathbf{a}, \mathbf{b})$. Experimental data (full black circle) are compared with ab initio phonon calculations after convolution with the instrumental resolution (red line). Calculated, individual phonon modes are reported in light grey (acoustic) and blue dotted line (optic). The energy scan at $\Gamma_{611}$ (f), for which the acoustic intensity is low, makes possible a direct observation of the low-lying optical intensities analysed using four Gaussian profiles centered at energies $E_{1}, E_{2}, E_{3}$ and $E_{4}$

(see Methods and Supplementary Notes 4 and 5). Longitudinal (LA) and transverse (TA) acoustic phonons were measured around the strong Bragg peak $(6,0,0)$. Figure 2 reports the $S(\mathbf{Q}, E)$ intensity distribution measured at $300 \mathrm{~K}$ around the zone centre $\Gamma_{600}$ for $\mathrm{LA}_{011}$ modes propagating along the $(0,1,1)$ direction (Fig. 2a) and for $\mathrm{TA}_{011}^{100}$ modes, propagating along $(0,1,1)$ and polarized along $(1,0,0)$ (Fig. $2 \mathrm{~b}$ ) on a neutron Time Of Flight
(TOF) spectrometer. Similarly to other clathrates ${ }^{27,29}$, for both transverse and longitudinal excitations, there are well-defined propagative acoustic excitations only in a limited range below a low-lying optical excitation. Figure 3 displays raw data (mapping of the phonon dispersions and constant Q-scans) measured on a neutron Triple Axis Spectrometer (TAS) at $300 \mathrm{~K}$ over a larger energy range, together with a comparison with simulated spectra 

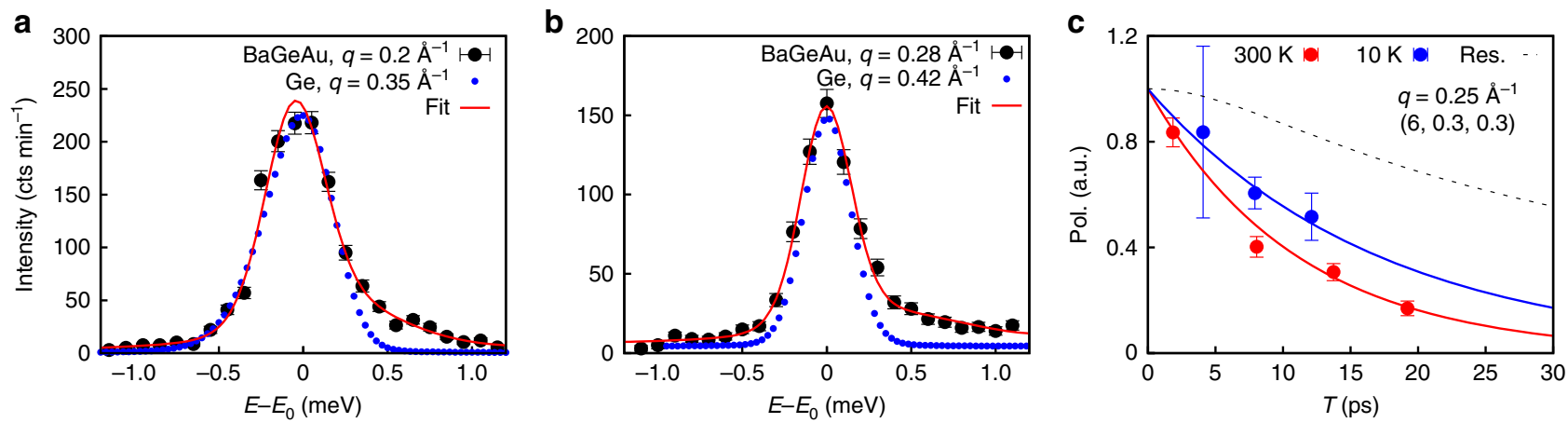

Fig. 4 Experimental measurements of transverse acoustic (TA) phonon lifetimes in $\mathrm{Ba}_{7.81} \mathrm{Ge}_{40.67} \mathrm{Au}_{5.33}\left(\mathrm{BaGeAu}\right.$ ). a, b Comparison between the $\mathrm{TA} \mathrm{b}_{011}^{100}$ phonons in BaGeAu clathrate (black points) and in pure Ge (blue dotted line) measured with constant $q$-scans, at $q=0.2 \AA^{-1}$ and $q=0.28 \AA^{-1}$ where their local group velocities are identical. Measurements were performed on the triple axis spectrometer (TAS) 2T@LLB with an effective resolution of $200 \mu \mathrm{eV}$. The Ge data represent directly the effective TAS resolution. Data are reported as a function of $E-E_{0}$, with $E_{0}$ the acoustic phonon mode energy. In $\mathbf{a}$, $E_{0}=$ $2.97 \mathrm{meV}(\mathrm{BaGeAu})$ and $E_{0}=5.72 \mathrm{meV}(\mathrm{Ge})$; in $\mathbf{b} E_{0}=4 \mathrm{meV}(\mathrm{BaGeAu})$ and $E_{0}=6.75 \mathrm{meV}(\mathrm{Ge})$. $\mathbf{c}$ Evolution of the neutron polarization as a function of $\tau_{\mathrm{NRSE}}$ for the $\mathrm{TA}_{011}^{100}$ phonon $\left(q=0.25 \AA^{-1}, E=3.8 \mathrm{meV}\right.$ ) measured with the Neutron Resonant Spin-Echo (NRSE) technic at $300 \mathrm{~K}$ (full red circle) and at $10 \mathrm{~K}$ (full blue circle) measured on the spectrometer IN22-ZETA@ILL. Data are corrected for the experimental resolution shown as a dotted line. The full blue and red lines are exponential fits to the data from which phonon lifetimes equal to 33(10) ps and 15(1) ps at 10 and $300 \mathrm{~K}$ are extracted. Vertical error bars in a-c are s.d. arising from counting statistics

convoluted with the experimental resolution for $\mathrm{TA}_{011}^{100}$ modes covering several Brillouin zones (all constant Q-scans are shown in the Supplementary Note 17). Similarly to other clathrates, the phonon spectra in $\mathrm{Ba}_{7.81} \mathrm{Ge}_{40.67} \mathrm{Au}_{5.33}$ can be separated into two energy regimes on either side of the lowest lying optic mode, labeled $E_{1}$. With an energy of $4.5 \mathrm{meV}$, this mode is lower than the one in $\mathrm{Ba}_{8} \mathrm{Ge}_{42.1} \mathrm{Ni}_{3.5}$ (measured at $5.5 \mathrm{meV}^{29}$, or in $\mathrm{Ba}_{8} \mathrm{Si}_{46}$ (observed at $7 \mathrm{meV}^{27}$ ), confirming ab initio predictions. It thus suppresses more acoustic states, in agreement with a lattice thermal conductivity at room temperature lower than in lighter transition metal (TM) substituted Ge clathrates without Ba-TM bonding (see Supplementary Note 5 and refs. ${ }^{41-44}$ ). Below $E_{1}$, well-defined acoustic modes are found which continuously disperse up to $E_{1}$, keeping a pure acoustic character only for energies lower than $3 \mathrm{meV}$, similarly to other clathrates $26,27,29$. Approaching $E_{1}$, the acoustic branches transfer their spectral weight to the optic modes, and bend over, their slope approaching zero at the Brillouin zone boundary (Figs. $2 b$ and $3 a, b)$. Above $4.3 \mathrm{meV}$ the phonon spectrum is dominated by optic modes, and can be modeled with four Gaussian distributions labeled $E_{1,2,3,4}$, describing the superposition of several weakly dispersive optical phonon branches and located at energies of 5, 7.5, 10 and $12 \mathrm{meV}$ respectively. As observed in other clathrates, depending on the dynamical range of the color mapping, the spectral weight transfer can be clear, as in Fig. 2 here, or can be hidden by an apparent going-on dispersion shared between the acoustic and the optic modes, as seen in Fig. 3a.

Our ab initio DFT simulation well reproduces the acoustic and optic phonon dispersions and the experimental intensity distribution (Fig. 3), a much constraining test. However, in order to achieve a good agreement we had to apply an energy scaling factor equal to 1.4 for the acoustic part $(E<6 \mathrm{meV})$ and 1.2 for modes at higher energies, similar to what was used for $\mathrm{Ba}_{8} \mathrm{Ge}_{42.1} \mathrm{Ni}_{3.5}{ }^{29}$. This might be ascribed to an underestimation of the $\mathrm{Ba} / \mathrm{Au}-\mathrm{Ge}$ interactions within the DFT approach.

$\mathrm{Ab}$ initio DFT simulations allow us to compute the contribution of the different sites to the signal: we can thus assign the four $E_{1,2,3,4}$ optical excitations respectively to $\mathrm{Ba} 2-, \mathrm{Au}-, \mathrm{Ge}-, \mathrm{Ba} 1 / \mathrm{Ge}-$ dominated vibrations, as shown by the partial vibrational density of states (Supplementary Note 5 and Supplementary Fig. 11). One should also notice that including the experimentally observed disorder into the simulation is expected to broaden the simulated optical excitations ${ }^{24}$.
Measurements of the phonon lifetimes in $\mathrm{Ba}_{7.81} \mathrm{Ge}_{40.67} \mathrm{Au}_{5.33}$. Quantitative measurements of acoustic phonon lifetimes are extremely challenging. The key point is the central role of the instrumental resolution function, which depends both on the wave vector $\mathbf{q}$ and the energy $E$ so that, for dispersive excitations, the observed peak width depends on the local curvature of the phonon dispersion $E(\mathbf{q})$ and thus on the local group velocity (see Supplementary Note 4, Supplementary Fig. 5 and refs. ${ }^{27,}{ }^{29}$ ). Benefiting from the large crystal volume, our first approach was to use a low-flux neutron TAS setup in order to achieve a high instrumental resolution equal to $200 \mu \mathrm{eV}$ (FWHM) for the $\mathrm{TA}_{011}^{100}$ modes, giving access to intrinsic broadenings larger than $50 \mu \mathrm{eV}$. Data collected at $300 \mathrm{~K}$ are shown in Fig. $4 \mathrm{a}$, b (more constant $q$ scans are shown in the Supplementary Figs. 7, 17). Although for phonons with $q<0.22 \AA^{-1}(E<3.3 \mathrm{meV})$, the peak width is resolution limited, a sizeable broadening of about $100 \mu \mathrm{eV}$ is observed for wave vectors between 0.25 and $0.32 \AA^{-1}$. At higher wave vectors, acoustic and optic peaks overlap, increasing the fit uncertainty on the phonon linewidths determination. To validate this result, we directly compare the intensity profile of the clathrate acoustic phonons with those measured under exactly the same conditions on a pure Germanium crystal, as shown Fig. 4a, b. Whereas both excitations are almost identical for the phonons measured at $q=0.2 \AA^{-1}$ (Fig. 4a) a sizeable broadening is observed at $0.28 \AA^{-1}$ in the clathrate (Fig. $4 \mathrm{~b}$ ), confirming that the measured widths for $q>0.22 \AA^{-1}(E>3.3 \mathrm{meV})$ are intrinsic.

To overcome the limited resolution of TAS experiments and measure lifetimes for phonons below $4 \mathrm{meV}$, the NRSE technique was used, in a configuration giving access to phonon lifetimes up to $66 \mathrm{ps}$, corresponding to an energy resolution of $20 \mu \mathrm{eV}^{22}$ (see Methods). Figure $4 \mathrm{c}$ shows a typical NRSE result from which the phonon lifetimes $\tau$ are extracted. In Table 1 , we report the measured values for the $\mathrm{TA}_{011}^{100}$ branch at $300 \mathrm{~K}$, at four phonon energies between 2 and $4.4 \mathrm{meV}$.

Figure 5 summarizes our results, showing the energy dependence of our TAS and NRSE measurements of the inverse lifetime $1 / \tau$ (and energy linewidth $\Gamma_{\mathrm{FWHM}}$ ) of acoustic phonons, and comparing them to published measurements in other complex crystalline and disordered materials in the same energy range. In our clathrate, $1 / \tau$ increases with energy up to $3.2 \mathrm{meV}$, where then it is roughly constant up to $4.4 \mathrm{meV}$. Interestingly, such a step-like behavior is also seen in the computed phonon density-of-states (p-DOS, black dotted line in Fig. 5). At the 
Table 1 Summary of the experimental $\mathrm{TA}_{011}^{100}$ individual phonons properties $\mathrm{Ba}_{7.81} \mathrm{Ge}_{40.67} \mathrm{Au}_{5.33}$

\begin{tabular}{llllllll}
$\boldsymbol{T}(\mathbf{K})$ & $\mathbf{q}(\mathbf{0}, \boldsymbol{\xi}, \boldsymbol{\xi})(\boldsymbol{r . l . u .})$ & $\mathbf{q}\left(\boldsymbol{A}^{-\mathbf{1}}\right)$ & $\boldsymbol{E}(\mathbf{m e V})$ & $\boldsymbol{\Gamma}_{\mathbf{F W H M}}(\boldsymbol{\mu} \mathbf{e V})$ & $\boldsymbol{\tau}(\mathbf{p s})$ & $\boldsymbol{V}_{\mathbf{G}}\left(\mathbf{m ~ s} \mathbf{s}^{-\mathbf{1}}\right)$ & $\boldsymbol{I}(\mathbf{n m})$ \\
\hline 300 & 0.143 & 0.11 & 2 & $25(2)$ & $82(7)$ & $16(1)$ & 2260 \\
300 & 0.24 & 0.19 & 3.2 & $87(7)$ & $15(1)$ & 1950 \\
300 & 0.30 & 0.25 & 3.8 & $96(12)$ & $14(1)$ & 1530 \\
300 & 0.36 & 0.29 & 4.4 & $24(10)$ & $55(17)$ & 2260 \\
10 & 0.143 & 0.11 & 2 & $40(13)$ & $33(11)$ & 1690
\end{tabular}

The columns report temperature $T(\mathrm{~K})$, the phonon wavevector $q$ in r.l.u. and in $\AA^{-1}$, the phonon energy $E$ in meV, the phonon FWHM width $\Gamma_{\mathrm{FWHM}}$ ( $\mu \mathrm{eV}$ ), the phonon lifetime $\tau$ in ps, the phonon group velocity in $\mathrm{m}^{-1} \mathrm{~s}^{-1}$ and the phonon mean free path $/ \mathrm{in} \mathrm{nm}$. The relationship between the phonon width in energy $\Gamma_{\mathrm{FWHM}}(\mu \mathrm{eV})$ and the lifetime $\tau(\mathrm{ps})$ is $\tau(\mathrm{ps})^{-1}=\frac{\Gamma_{\mathrm{FWHM}}}{2 \tau}=0.7592 .10^{-3} . \Gamma_{\mathrm{FWHM}}(\mu \mathrm{eV})^{21,27}$

lowest energy value, $E=2 \mathrm{meV}$, a lifetime of 53(4) ps is found which, with the group velocity at this energy, corresponds to a phonon mean free path of $120 \mathrm{~nm}$. At $E=3.2 \mathrm{meV}, 3.8$ and 4.4 $\mathrm{meV}$, the phonon lifetimes are of the order $15 \mathrm{ps}$, and the phonon mean free paths are 31,25 and $21 \mathrm{~nm}$, respectively, i.e. more than 10 times larger than the unit cell $(a=1.08 \mathrm{~nm})$. The measured lifetimes are much smaller than in pure Ge where, from the measured $\tau$ about $66 \mathrm{ps}$ at $9.8 \mathrm{meV}^{45}$, we can estimate $\tau$ about $660 \mathrm{ps}$ at $3 \mathrm{meV}$. Our results are also compared in Fig. 5 to previously obtained data in other thermoelectrics such as $\mathrm{PbTe}^{17-19}$ or $\mathrm{AgSbTe}_{2}{ }^{19,42}$ and amorphous Silica ${ }^{46-48}$. For the clathrate $\mathrm{Ba}_{7.81} \mathrm{Ge}_{40.67} \mathrm{Au}_{5.33}$ and in the energy range $2-4 \mathrm{meV}$ we find much larger lifetimes than in all these compounds.

It is also interesting to compare the acoustic mean free path here found with the ones of the optical excitations $E_{2}, E_{3}$ and $E_{4}$, whose experimental lifetime is of the order of $1 \mathrm{ps}$, assuming that a single excitation contribute to each of these modes (Supplementary Note 4). Optical excitations are almost dispersionless or weakly dispersing (Fig. 3), so that their mean free path is extremely short. For the excitation $E_{2}$ its maximum value is equal to $1.5 \mathrm{~nm}$, i.e., more than one order of magnitude lower than for the $4.4 \mathrm{meV}$ acoustic excitations.

Finally, we have investigated the temperature dependence of the phonon lifetime, by repeating the neutron TAS and NRSE measurements at $10 \mathrm{~K}$ at the same conditions during the same experiments. Figure 6 compares the neutron TAS intensity profiles measured at $300 \mathrm{~K}$ and $10 \mathrm{~K}$ for those phonons for which an intrinsic linewidth was found. With the TAS, the sensitivity being limited at $50 \mu \mathrm{eV}$ (Supplementary Note 4 and Supplementary Fig. 5), we cannot resolve any temperature dependence of the phonon linewidth, but we demonstrate the absence of a strong shift of the phonon energies, confirming previous results at $300 \mathrm{~K}$ in clathrates 27,29 , which show that the guest phonon modes are coherently coupled with the host lattice without anharmonicity. NRSE reveals a temperature effect of the phonon lifetime at $E=$ $3.8 \mathrm{meV}$, which decreases from $33(10) \mathrm{ps}$ at $10 \mathrm{~K}$ to $15(1) \mathrm{ps}$ at $300 \mathrm{~K}$ (Fig. 5 and Table 1), while no temperature effect is observed at $2 \mathrm{meV}$, whose value at $300 \mathrm{~K}$ is already at the limit of detection.

A simple phenomelogical model for the thermal conductivity in complex materials. Using these experimental results we can calculate the lattice thermal conductivity $\kappa_{\mathrm{L}}$, which, in the framework of the relaxation-time approximation of the Boltzmann equation, is given by:

$$
\kappa_{\mathrm{L}}=\frac{1}{3} \int_{0}^{\omega_{\max }} C_{\mathrm{v}}(\omega) v^{2}(\omega) \tau(\omega) \rho(\omega) \mathrm{d} \omega
$$

where $C_{\mathrm{v}}(\omega), v(\omega)=\partial \omega / \partial q, \tau(\omega)$ and $\rho(\omega)$ are the phonon specific heat per unit volume, velocity, lifetime and phonon density of states respectively.
Instead of considering the $3 \times 54$ phonon modes we assume, as was proposed for complex dielectrics ${ }^{49-51}$, that only the three acoustic branches contribute to $\kappa_{\mathrm{L}}$. Indeed, above the acoustic cutoff frequency, the measured optical excitations are both weakly dispersive and rather broad with lifetimes much shorter than the one of acoustic modes, thus leading to minor contributions to the lattice thermal conductivity and to major contributions to the heat capacity. The cutoff frequency for a given acoustic branch, $\omega_{\max }$, is such that $\int_{0}^{\omega_{\max }} \rho(\omega) \mathrm{d} \omega=1$ and is equal to $4.7 \mathrm{meV}$ for TA modes, in agreement with the experimental dispersion relation (Figs. 2, 3).

We then assume a phenomenological energy dependence of the acoustic phonon lifetimes: for $\hbar \omega<3.2 \mathrm{meV}$, it mimics the one of the p-DOS $(\omega)$ (black dotted line in Fig. 5), $\tau(\omega) \propto \mathrm{p}-\operatorname{DOS}(\omega)$, while for $3.2 \mathrm{meV} \leq \hbar \omega<\hbar \omega_{\max }$, the lifetime is fixed at $16 \mathrm{ps}$. The acoustic phonon dispersions are assumed isotropic, following experimental results here and in other clathrates ${ }^{29}$. With these assumptions, Eq. 1 yields at room temperature $\kappa_{\mathrm{L}}=1.2 \mathrm{~W} \mathrm{~m}^{-1}$ $\mathrm{K}^{-1}$ in very good agreement with the experimental value $\left(1.1 \mathrm{~W} \mathrm{~m}^{-1} \mathrm{~K}^{-1}\right)$.

This confirms previous studies showing that in complex dielectric crystals $\kappa_{\mathrm{L}}$ is well approximated by considering only the acoustic phonons, even though the optic modes mainly contribute to the heat capacity, here more than $98 \%$ (the DulongPetit limit gives $C_{\mathrm{DP}}^{\mathrm{op}}=259.47 \mathrm{~mJ} \mathrm{~g}^{-1} \mathrm{~K}^{-1}$ while $C_{\mathrm{DP}}^{\mathrm{ac}}=4.91 \mathrm{~mJ} \mathrm{~g}^{-1}$ $\mathrm{K}^{-1}$ ), and provide scattering channels for acoustic phonons $3,21,52,53$. This approximation works especially well in guest-host materials where atomic masses and chemical bonds differ substantially. We can thus understand thermal transport in clathrates as essentially due to acoustic modes, propagating in a small energy range, limited by the lowest-lying optic mode, and on distances of a few tens of nanometers. In agreement with recent studies in clathrates, guest modes act as a 'low band pass' filter for acoustic phonons 27,29 . This model also works in intermetallic quasicrystals and their periodic approximants, where an almost temperature independent thermal conductivity in the $\mathrm{W} \mathrm{m}^{-1} \mathrm{~K}^{-1}$ range is observed. Here, the energy cutoff limiting the acoustic regime is $5-7 \mathrm{meV}^{50,54,55}$ resulting in mean free paths of $20-30 \mathrm{~nm}$ for phonon wave-vectors below $0.3 \AA^{-1}$, in agreement with recent estimations ${ }^{53}$. Note that a valid firstorder approximation of the thermal conductivity can be obtained in the classical kinetic gas limit of Eq. 1, i.e., the Debye model, considering only the three acoustic phonons and a constant phonon lifetime (Supplementary Note 6). The energy-dependent acoustic contribution can be estimated looking at the accumulated thermal conductivity $\kappa_{\mathrm{L}}(\omega)=\frac{1}{3} \int_{0}^{\omega} C_{\mathrm{v}}(\omega) v^{2}(\omega) \tau(\omega) \rho(\omega) \mathrm{d} \omega$. About $50 \%$ of the thermal conductivity is carried by phonon in the range $0-2 \mathrm{meV}$, or the range $2-4.5 \mathrm{meV}$ (Supplementary Note 6 and Supplementary Fig. 15). The acoustic phonons lying in the linear part of the dispersion, with $E<2 \mathrm{meV}$ and the largest velocities, have a larger relative weight at lower 


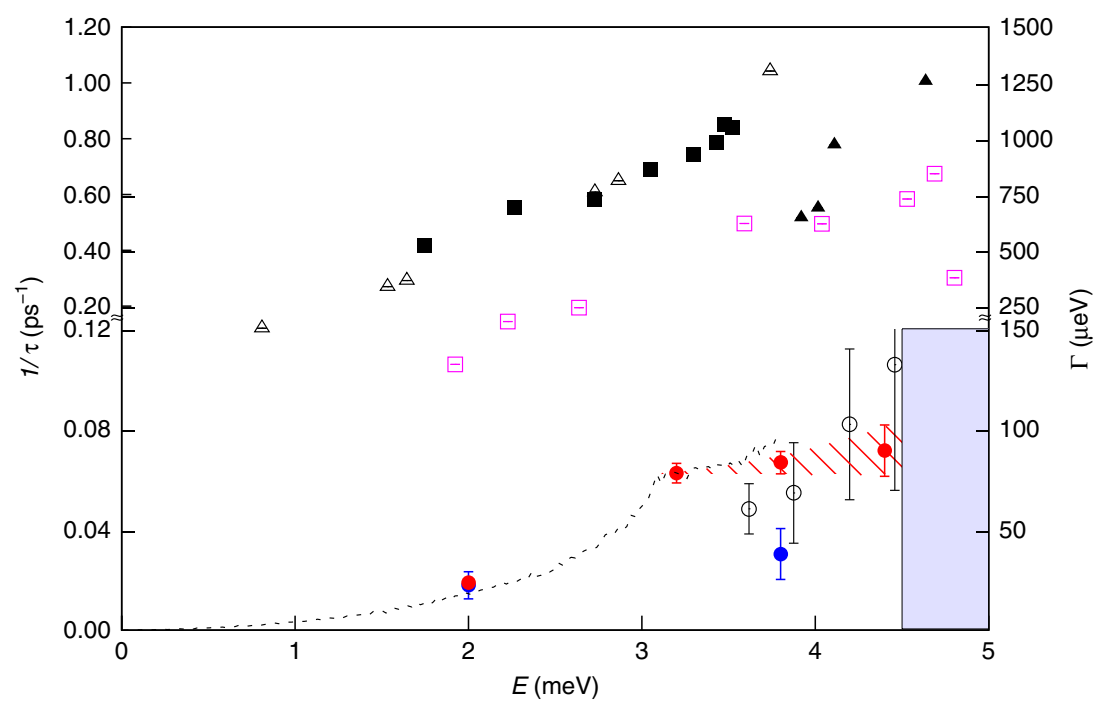

Fig. 5 Energy and temperature dependences of transverse acoustic (TA) phonon lifetimes in $\mathrm{Ba}_{7.81} \mathrm{Ge}_{40.67} \mathrm{Au}_{5.33}$. $\mathrm{TA}_{011}^{100}$ inverse phonon lifetimes (left) and energy Full Width at Half Maximum (FWHM, right) as determined from measurements on the neutron triple axis spectrometer (TAS, black circles) and the neutron resonant spin-echo (NRSE) spectrometer as a function of energy at $300 \mathrm{~K}$ (full red circles) and at $10 \mathrm{~K}$ (full blue circles). Above $4.5 \mathrm{meV}$ the extraction of a single acoustic phonon mode lifetime is no longer possible due to the proximity of a low lying optical excitation (blue shaded area). Vertical error bars are standard deviation obtained by error propagation during the fitting procedure. The dotted line shows the rescaled calculated phonon density of state ( $\mathrm{p}-\mathrm{DOS}(\omega)$, in $\mathrm{meV}^{-1}$; see Supplementary Note 5) which is truncated at $E=3.8 \mathrm{meV}$ due to van Hove singularities. The calculated phonon lifetime from DFT (see text) for the $\mathrm{TA}_{011}^{100}$ mode is shown as (empty pink squares). Previous experimental determination of acoustic phonon lifetimes in the same energy range are reported for PbTe (full black squares, Delaire et al. ${ }^{17}$ ), AgSbTe 2 (empty triangles, Ma et al. ${ }^{19,}{ }^{21}$ ) and amorphous Silica (full black triangles, Baldi et al. ${ }^{48}$ )

temperature. These phonons, with energies lower than the energy difference between the lowest optical modes, $E_{2}^{\text {optic }}-E_{1}^{\text {optic }} \sim 2$ $\mathrm{meV}$, are less affected by the three-phonon scattering processes involving these optical modes (acoustic + optic (1) <-> optic(2)), as determined by the energy and momentum conservation laws ${ }^{3}$.

Theoretical simulations of the three-phonon anharmonic scattering processes in the clathrate $\mathrm{BaGeAu}$. Identifying what are the underlying mechanisms responsible for the observed phonon lifetimes requires an analysis of both their energy and temperature dependence. There are four possible phonon scattering processes that can be considered: three-phonon anharmonic processes, scattering on defects, scattering on electrons and scattering on domain boundaries ${ }^{56}$. On one hand our experimental results do not allow to uniquely identify the different processes, on the other hand very little has been done on simulations of structurally complex and disordered materials as explained in the introduction. For instance, models for disorder are only available for the so-called mass defects in simple structures as $\mathrm{Ge}^{11,13,14}$ and DFT electron-phonon calculations have only been achieved recently for $\mathrm{Si}^{57}$. Simple anharmonic or disordered models can neither account for the observed relatively small temperature dependence nor for the rather unexpected frequency dependence of the phonon lifetimes.

As a first attempt to computationally tackle these issues, we have however calculated the three-phonon scattering rates and corresponding phonon lifetime contributions using DFT calculations and the method recently proposed and applied by Tadano et al. ${ }^{40}$ on similar clathrates. We performed the simulation on a single unit cell with the ideal composition $\mathrm{Ba}_{8} \mathrm{Ga}_{40} \mathrm{Au}_{6}$ without any disorder, i.e., $\mathrm{Ba}$ atoms are located at the cage centre and there is no $\mathrm{Au} / \mathrm{Ge}$ disorder (Supplementary Note 5). Indeed, current computation limitations do not allow for calculations on a $2 \times 2 \times 2$ supercell that would be required for describing the observed disorder.
Figure 7 reports the temperature dependence of the experimental and simulated phonon lattice thermal conductivity, while phonon lifetimes are presented in the Supplementary Note 5. In order to roughly reproduce the low $T$ part of the observed thermal conductivity $(T<50 \mathrm{~K})$, we have introduced boundary effect, with grain size of $500 \mathrm{~nm}$ as shown by the blue line in Fig. 7. This size is likely too small, and other mechanisms (electron-phonon and defects) should be included to explain the low $T$ behavior. However, this is beyond the scope of the present work, where we concentrate on the high temperature dependence, for $T$ larger than $50 \mathrm{~K}$.

Our simulations are qualitatively and quantitatively very similar to the results obtained by Tadano et al. for $\mathrm{Ba}_{8} \mathrm{Ga}_{16} \mathrm{Ge}_{30}$ in ref. ${ }^{40}$ and display a number of discrepancies with our experimental results. First of all, the calculated room temperature lifetimes and lattice thermal conductivities are smaller than the experimental values by a factor 3 to 5 as exemplified in Figs. 5, 7 .

A similar discrepancy is actually also observed in the simulation of Tadano et al. for $\mathrm{Ba}_{8} \mathrm{Ga}_{16} \mathrm{Ge}_{30}$ in ref. ${ }^{40}$ if temperatures larger than $100 \mathrm{~K}$ are considered (at $300 \mathrm{~K}$ the experimental value is equal to $1.3 \mathrm{~W} \mathrm{~m} \mathrm{~W}^{-1} \mathrm{~K}^{-1}$ in ref. ${ }^{58}$ whereas the simulation in ref. ${ }^{40}$ yields $\left.0.4 \mathrm{~W} \mathrm{~m}^{-1} \mathrm{~K}^{-1}\right)$. Note that a phonon lifetime equal to $2-3 \mathrm{ps}$, as predicted by the simulation at $4 \mathrm{meV}$, would be easily detected experimentally. Moreover, simulations carried out on simple models for the observed disorder further decrease the phonon lifetime and thermal conductivity, as expected (Supplementary Note 5, Supplementary Figs. 12 and 13), thus increasing the disagreement with the experiment.

The second point of disagreement is the temperature dependence of both phonon lifetimes and thermal conductivity, which in simulations scale both as $1 / T$ for $T$ larger than $40 \mathrm{~K}$, simply reflecting Bose-Einstein statistics, while experimentally display a very weak dependence between 50 and $300 \mathrm{~K}$ (Fig. 7).

It is worth underlying, however, that despite all these points of disagreement, simulations rather well reproduce the experimentally observed energy dependence of the inverse lifetime, which in 

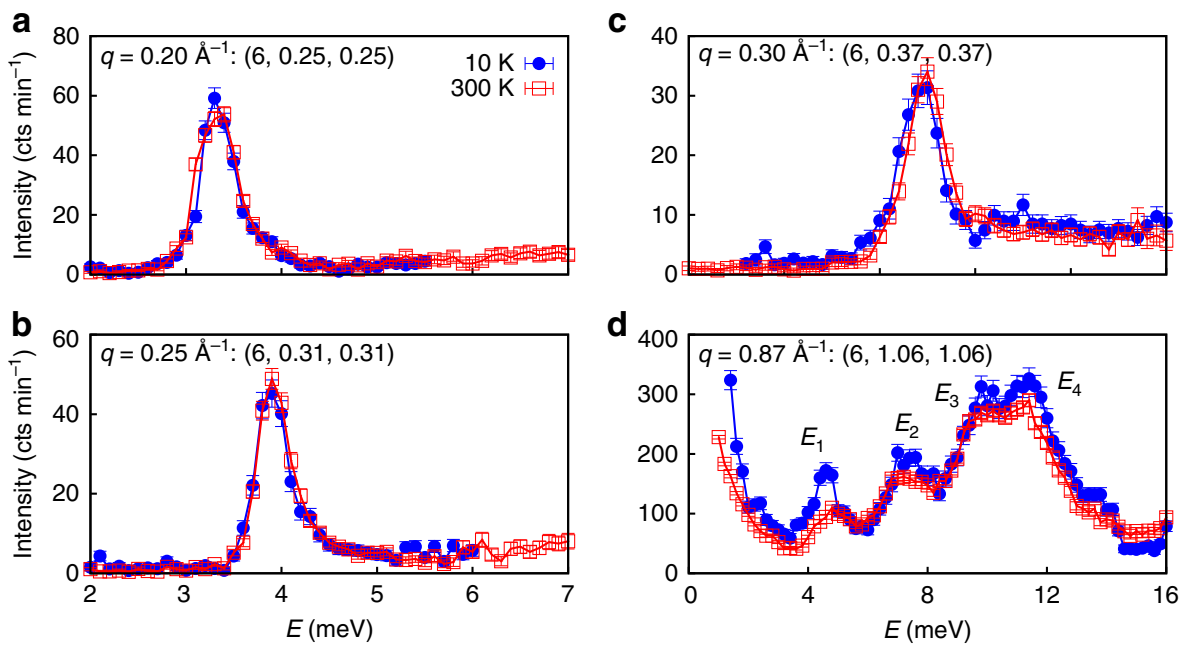

Fig. 6 Transverse acoustic (TA) phonons profiles measured at 10 and $300 \mathrm{~K}$ in $\mathrm{Ba}_{7.81} \mathrm{Ge}_{40.67} \mathrm{Au}_{5.33}$. a-c Comparison between constant $q$-scans of the TA ${ }_{011}^{100}$ branch measured at $10 \mathrm{~K}$ (full blue circles) and $300 \mathrm{~K}$ (red squares) on the triple axis spectrometer (called 2T@LLB) with an effective resolution of 200 peV. d Similar comparison between scans with a larger resolution of $1.2 \mathrm{meV}$ measured at the wave vector $(6,1.06,1.06)$ close to the centre of the BZ where the four optic phonon modes, labeled $E_{1,2,3,4}$ in the text, are clearly visible $(\mathbf{a}, \mathbf{b})$. In a-d, the intensities are corrected for the Bose population factor. Vertical error bars are s.d. from counting statistics

the long wavelength limit roughly scales with $\omega^{2}$ (see Fig. 5 and Supplementary Note 5).

The origin of the discrepancy on the temperature dependence and the absolute values of phonon lifetimes and thermal conductivity needs thus to be sought elsewhere, and requires further measurements of both temperature and energy dependence of phonons lifetime, coupled to atomistic or molecular dynamics simulations taking into account all the present results, as well as a possibly temperature-dependent force field.

Indeed, the $1 / T$ decay in simulations is related to the Bose-Einstein phonon population factor, with the assumption that the force field does not change with temperature (see ref. ${ }^{9}$ ). If this assumption is released, the temperature dependence of the simulated thermal conductivity could be different.

Concerning the absolute value of phonon lifetimes and thermal conductivity, the current phonon Umklapp simulations might underestimate them. Indeed is worth noticing that a slight change of the lattice parameter used in simulations can strongly affect them, as shown by Tadano et al. ${ }^{10}$ Going from the relaxed $(1.10 \mathrm{~nm})$ to the experimental lattice parameter $(1.08 \mathrm{~nm})$ leads to a factor 4 increase in the phonon thermal conductivity and average phonon lifetimes (see Supplementary Note 5 and Supplementary Figs. 13, 14). This is most likely associated with the underestimation of the phonon energies by the DFT calculation as already pointed out before.

Finally, the structural complexity and the large chemical disorder are not fully taken into account in the DFT simulation, for they likely are related to temperature independent two phonons scattering processes.

To summarize, our experimental results point to a profound disagreement with state of the art simulations and ask for future advanced calculations, better describing the results of our detailed structural analysis, including the large disorder, as well as a non-trivial temperature dependence of the 3-phonons scattering. In particular, molecular dynamics simulations as carried out by Tse et al. in clathrate hydrate $e^{35,59}$ or in Ge clathrate using model Hamiltonian as in refs. ${ }^{28,60}$ allows to handle large system size ${ }^{61}$. We believe that the data provided here will give a robust starting point for such studies.

\section{Discussion}

Our main result is the first accurate measurement of the acoustic phonon mean free path in a complex crystalline system,

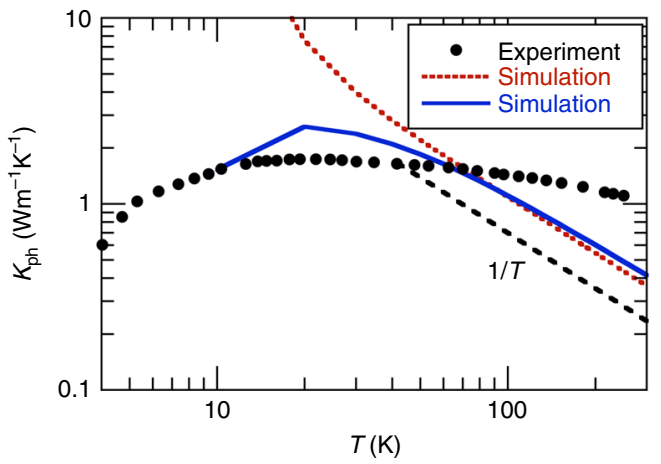

Fig. 7 Lattice thermal conductivity in $\mathrm{Ba}_{7.81} \mathrm{Ge}_{40.67} \mathrm{Au}_{5.33}$. Comparison between the experiment (full circles) and the anharmonic three phonon simulation (red dotted line) carried out using the experimental lattice parameter. The blue solid line includes boundary effect with a size of 500 $\mathrm{nm}$. The dashed black line indicates the 1/T decay. Other scattering mechanisms (defects, electron-phonon) needs to be included at temperature lower than $50 \mathrm{~K}$

specifically $\mathrm{Ba}_{7.81} \mathrm{Ge}_{40.67} \mathrm{Au}_{5.33}$. To summarize our findings, at $300 \mathrm{~K}$, the mean free path goes from 120 to $20 \mathrm{~nm}$ as the phonon wavevector and energy go from 0.1 to $0.3 \AA^{-1}$ and 2 to $4.4 \mathrm{meV}$, respectively. As already pointed out, this is much larger than any other previously experimentally accessed phonon mean-free path in similar systems and in the energy range $2-4 \mathrm{meV}$.

It is thus interesting to compare these results with other thermoelectric systems for which DFT simulations give a good agreement with experiment, namely the $\mathrm{PbTe}^{8}$ system with two atoms per unit cell and the $\mathrm{Bi}_{2} \mathrm{Te}_{3}{ }^{9}$ one, with five atoms per unit cell. These two systems have room temperature thermal conductivities equal to 2 and $1.5 \mathrm{~W} \mathrm{~m}^{-1} \mathrm{~K}^{-1}$, respectively and in both cases simulations predict that about $80 \%$ of the heat is carried by acoustic modes.

$\mathrm{PbTe}$ exhibits experimental acoustic phonon lifetimes much shorter than our case, despite a similar thermal conductivity at room temperature. It is important to note however that recent DFT calculations in $\mathrm{PbTe}^{8}$, in reasonable agreement with inelastic neutron scattering data ${ }^{18}$, report simulated acoustic phonon lifetimes roughly scaling with $1 / \omega^{2}$, and with a value of the order 
of $10 \mathrm{ps}$ for TA modes with an energy of $4 \mathrm{meV}$ at $300 \mathrm{~K}$, smaller but close to our findings, and a lifetime of about $1 \mathrm{ps}$ for optic modes. It results that most of the heat carrying phonons have simulated mean free paths in the range 2 to $20 \mathrm{~nm}$ at $300 \mathrm{~K}$, similarly to our case.

The case of $\mathrm{Bi}_{2} \mathrm{Te}_{3}$ seems much more similar to ours. Indeed in this system the acoustic regime is restricted to the range $0-6 \mathrm{meV}$, followed by weakly dispersive optical excitations in the range 6-10 $\mathrm{meV}$ and dispersionless optical modes in the range 10-16 $\mathrm{meV}$. In fact this system bears some similarities with our clathrate, with an acoustic energy cut-off at about the same value of 6 $\mathrm{meV}$, despite a sound velocity about 1.7 times smaller. Moreover, the heat carrying phonons have mean free paths in the range 7 to $70 \mathrm{~nm}$, again very similar to our findings (see ref. ${ }^{62}$ ).

Despite the similarities between these and our system, in $\mathrm{PbTe}$ and $\mathrm{Bi}_{2} \mathrm{Te}_{3}$ the thermal conductivity changes as $1 / T$ at high temperature, in perfect agreement with a dominating rule of three -phonon processes, well reproduced in DFT simulations ${ }^{8}$. Moreover in the clathrate system the phase space available is also somewhat smaller than in the above two, since the heat carrying phonons only exist in the acoustic regime for the $0-4 \mathrm{meV}$ energy range.

In summary, we have performed a quantitative measurement of the acoustic phonon lifetimes in a clathrate $\mathrm{Ba}_{7.81} \mathrm{Ge}_{40.67} \mathrm{Au}_{5.33}$ crystal at 300 and $10 \mathrm{~K}$. We find propagative heat carriers with long lifetime, travelling distances from more than hundred to twenty unit cells for wave-vectors between 0.1 and $0.3 \AA^{-1}$. Considering only the low-energy acoustic phonons and the experimentally determined phonon lifetimes allows us to reproduce the room temperature lattice thermal conductivity. Our results provide a picture of thermal transport in these systems and dramatically underline the inability of state of the art simulations to reproduce the observed phonons lifetime and thermal conductivity, asking for theoretical developments, for which we provide here a crucial experimental input.

\section{Methods}

Material synthesis and characterization. The single crystal of $\mathrm{Ba}_{7.81} \mathrm{Ge}_{40.67} \mathrm{Au}_{5.33}$ for neutron measurements was grown by the Bridgman technique ${ }^{41}$. Its crystal structure was characterized by single-crystal X-ray diffraction. The bulk mosaicity, as determined by hard X-ray and neutron Larmor diffraction, is less than 1' (Supplementary Note 1). The thermal conductivity was measured between 4 and $300 \mathrm{~K}$ (Supplementary Note 3).

Thermal conductivity measurement. The thermal conductivity measurements were carried out in a home-made device using a steady state heat flow method in the temperature range $4 \mathrm{~K}<T<300 \mathrm{~K}$. The temperature gradient along the sample was applied by a small-resistance-chip heater with a room-temperature resistance of $120 \Omega$. The temperature gradient along the sample was determined by a differential $\mathrm{Au} / 0.07 \% \mathrm{Fe}$ vs. Chromel thermocouple and the absolute temperature was measured by a Ge resistor below $40 \mathrm{~K}$ and a Pt resistor from 40 to $300 \mathrm{~K}$. Three concentric radiation shields were mounted around the sample in order to reduce the radiation effect. The uncertainty of the thermal conductivity measurement is $5 \%$. Nevertheless, data above $\sim 100 \mathrm{~K}$ were affected by the radiation. The radiation effect was subtracted by using thermal conductivity data measured above $80 \mathrm{~K}$ by means of the $3 \omega$ technique in a commercial nitrogen flow cryostat from Cryovac. This is an ac technique which heats the sample locally and thus reduces errors caused by radiation at room temperature and below to a negligible level ${ }^{63}$. A narrow metal line $(20 \mu \mathrm{m}$ wide and $1 \mathrm{~mm}$ long) serves as both the heater and the thermometer. To avoid electrical contact between heater and sample the polished sample surface is covered with a thin layer of $\mathrm{SiO}_{2}$ by chemical vapour deposition $^{64}$. More details on used $3 \omega$ setup can be found in ref. ${ }^{64}$.

Transmission electron microscopy. The Dresden Grand ARM (double corrected JEM-ARM300F) microscope was used for aberration-corrected TEM and STEM imaging at $300 \mathrm{kV}^{65}$.

Neutron scattering. Neutron measurements were performed on time-of-flight (TOF) and triple axis spectrometers (TAS) at the Institut Laue Langevin (IN5@ILL and IN22@ILL, Grenoble, France) and at the Laboratoire Léon Brillouin (2T@LLB, Saclay, France; for instruments and analysis details see Supplementary Note 4). The $\mathrm{LA}$ and TA dispersion and phonon lifetimes in $\mathrm{Ba}_{7.81} \mathrm{Ge}_{40.67} \mathrm{Au}_{5.33}$ were measured around the strong Bragg peak (600) at $Q=3.6 \AA^{-1}$ in the scattering plane [100]-
[011]. The instrumental resolution model when measuring dispersive excitation, calculated within the "affit" program, was carefully checked using a Ge single crystal (Supplementary Note 4). The experimental data have been then fitted using an excitation model (Damped Harmonic Oscillator: DHO) convoluted by the instrumental resolution (as detailed in ref. ${ }^{29}$ ). The NRSE technique, on the TAS spectrometer IN22 at ILL ("ZETA" option), was used to determine phonon lifetimes up to $66 \mathrm{ps}$ (for details see Supplementary Note 4).

Theoretical calculations. DFT ab initio calculations with the VASP and PHONON codes were used to simulate the p-DOS, phonon dispersion, INS phonon cross section and the $\mathrm{Au} / \mathrm{Ge}$ disordered configurations on supercells with sizes $2 \times$ $2 \times 2$ (Supplementary Notes 2,5). Simulations of phonon lifetimes in $\mathrm{Ba}_{8} \mathrm{Ge}_{40} \mathrm{Au}_{6}$ by considering cubic anharmonicities and the resulting phonon-phonon scattering, were performed using the ALAMODE code (Supplementary Note 5).

Data availability. The authors declare that the data supporting the findings of this study are available within the article and its Supplementary Information file.

Received: 16 December 2016 Accepted: 10 July 2017

Published online: 08 September 2017

\section{References}

1. Snyder, G. J. \& Toberer, E. S. Complex thermoelectric materials. Nat. Mater. 7, 105-114 (2008).

2. Toberer, E. S., Zevalkink, A. \& Snyder, G. J. Phonon engineering through crystal chemistry. J. Mater. Chem. 21, 15843 (2011).

3. Bouyrie, Y. et al. From crystal to glass-like thermal conductivity in crystalline minerals. Phys. Chem. Chem. Phys. 17, 19751-19758 (2015).

4. Minnich, A. J. Advances in the measurement and computation of thermal phonon transport properties. J. Phys. Condens. Matter. 27, 53202 (2015).

5. Broido, D. A., Malorny, M., Birner, G., Mingo, N. \& Stewart, D. A. Intrinsic lattice thermal conductivity of semiconductors from first principles. Appl. Phys. Lett. 91, 231922 (2007)

6. Esfarjani, K., Chen, G. \& Stokes, H. T. Heat transport in silicon from firstprinciples calculations. Phys. Rev. B 84, 85204 (2011).

7. Chaput, L. Direct solution to the linearized phonon Boltzmann equation. Phys Rev. Lett. 110, 265506 (2013).

8. Tian, Z. et al. Phonon conduction in $\mathrm{PbSe}, \mathrm{PbTe}$, and $\mathrm{PbTe}_{1-\mathrm{x}} \mathrm{Se}_{\mathrm{x}}$ from firstprinciples calculations. Phys. Rev. B 85, 184303 (2012).

9. Hellman, O. \& Broido, D. A. Phonon thermal transport in $\mathrm{Bi}_{2} \mathrm{Te}_{3}$ from first principles. Phys. Rev. B 90, 134309 (2014).

10. Tadano, T., Gohda, Y. \& Tsuneyuki, S. Anharmonic force constants extracted from first-principles molecular dynamics: applications to heat transfer simulations. J. Phys. Condens. Matter. 26, 225402 (2014).

11. Klemens, P. G. Thermal resistance due to point defects at high temperatures. Phys. Rev. 119, 507 (1960).

12. Li, W., Carrete, J., Katcho, N. A. \& Mingo, N. ShengBTE: a solver of the Boltzmann transport equation for phonons. Comp. Phys. Commun. 185, 1747-1758 (2014).

13. Abeles, B. Lattice thermal conductivity of disordered semiconductor alloys at high temperatures. Phys. Rev. 131, 1906 (1963).

14. Tamura, S. Isotope scattering of dispersive phonons in Ge. Phys. Rev. B 27 , 858-866 (1983)

15. Garg, J., Bonini, N., Kozinsky, B. \& Marzari, N. Role of disorder and anharmonicity in the thermal conductivity of silicon-germanium alloys: a firstprinciples study. Phys. Rev. Lett. 106, 45901 (2011).

16. Nilsson, G. \& Nelin, G. Phonon dispersion relations in Ge at 80 K. Phys. Rev. B 3, 364 (1971)

17. Delaire, O. et al. Giant anharmonic phonon scattering in PbTe. Nat. Mater. 10, 614-619 (2011).

18. Shiga, T. et al. Microscopic mechanism of low thermal conductivity in lead telluride. Phys. Rev. B 85, 155203 (2012).

19. Ma, J. et al. Phonon scattering rates and atomic ordering in $\mathrm{Ag}_{1-\mathrm{x}} \mathrm{Sb}_{1+\mathrm{x}} \mathrm{Te}_{2+\mathrm{x}}$ $(x=0,0.1,0.2)$ investigated with inelastic neutron scattering and synchrotron diffraction. Phys. Rev. B 90, 134303 (2014).

20. Voneshen, D. J. et al. Suppression of thermal conductivity by rattling modes in thermoelectric sodium cobaltate. Nat. Mater. 12, 1028-1032 (2013).

21. Ma, J. et al. Glass-like phonon scattering from a spontaneous nanostructure in $\mathrm{AgSbTe}_{2}$. Nat. Nanotechnol 8, 445-451 (2013).

22. Aynajian, P. et al. Energy gaps and Kohn anomalies in elemental superconductors. Science 319, 1509-1512 (2008).

23. Takabatake, T., Suekuni, K., Nakayama, T. \& Kaneshita, E. Phonon-glass electron-crystal thermoelectric clathrates: Experiments and theory. Rev. Mod. Phys. 86, 669 (2014). 
24. H. Lee, C., Hase, I., Sugawara, H., Yoshizawa, H. \& Sato, H. Low-lying optical phonon modes in the filled skutterudite $\mathrm{CeRu}_{4} \mathrm{Sb}_{12}$. J. Phys. Soc. Japan 75, 123602 (2006).

25. Koza, M. M. et al. Breakdown of phonon glass paradigm in La-and Ce-filled $\mathrm{Fe}_{4} \mathrm{Sb}_{12}$ skutterudites. Nat. Mater. 7, 805-810 (2008).

26. Christensen, M. et al. Avoided crossing of rattler modes in thermoelectric materials. Nat. Mater. 7, 811-5 (2008).

27. Pailhès, S. et al. Localization of Propagative Phonons in a Perfectly Crystalline Solid. Phys. Rev. Lett. 113, 25506 (2014).

28. Schopf, D., Euchner, H. \& Trebin, H.-R. Effective potentials for simulations of the thermal conductivity of type-I semiconductor clathrate systems. Phys. Rev. B 89, 214306 (2014)

29. Euchner, H. et al. Phononic filter effect of rattling phonons in the thermoelectric clathrate $\mathrm{Ba}_{8} \mathrm{Ge}_{40+\mathrm{x}} \mathrm{Ni}_{6-\mathrm{x}}$. Phys. Rev. B 86, 224303 (2012).

30. Keppens, V. et al. Localized vibrational modes in metallic solids. Nature 395, 876-878 (1998)

31. Christensen, S. et al. 'Glass-like' thermal conductivity gradually induced in thermoelectric $\mathrm{Sr}_{8} \mathrm{Ga}_{16} \mathrm{Ge}_{30}$ clathrate by off-centered guest atoms. J. Appl. Phys. 119, 185102 (2016).

32. Kamitakahara, W. A. \& Brockhouse, B. N. Vibrations of a mixed crystal: Neutron scattering from $\mathrm{Ni}_{55} \mathrm{Pd}_{45}$. Phys. Rev. B 10, 1200 (1974).

33. Maradudin, A. A. \& Califano, S. Theory of anharmonic processes in crystals with isotopic impurities. Phys. Rev. B 48, 12628 (1993).

34. Tse, J. S. et al. Anharmonic motions of $\mathrm{Kr}$ in the clathrate hydrate. Nat. Mater. 4, 917-921 (2005)

35. English, N. \& Tse, J. Mechanisms for thermal conduction in methane hydrate. Phys. Rev. Lett. 103, 015901 (2009).

36. Chaput, L., Bourgeois, J., Prytuliak, A., Koza, M. M. \& Scherrer, H. Simple view of the $\mathrm{Mg}_{2} \mathrm{Si}_{1-\mathrm{x}} \mathrm{Sn}_{\mathrm{x}}$ phonon spectrum: Sn resonances and mean field. Phys. Rev. B 91, 64304 (2015).

37. Li, W. \& Mingo, N. Ultralow lattice thermal conductivity of the fully filled skutterudite $\mathrm{YbFe}_{4} \mathrm{Sb}_{12}$ due to the flat avoided-crossing filler modes. Phys. Rev. B 91, 144304 (2015).

38. English, N., Tse, J. \& Carey, D. Mechanisms for thermal conduction in various polymorphs of methane hydrate. Phys. Rev. B 80, 134306 (2009).

39. Carrete, J., Li, W., Mingo, N., Wang, S. \& Curtarolo, S. Finding unprecedentedly low-thermal-conductivity half-Heusler semiconductors via high-throughput materials modeling. Phys. Rev. X 4, 11019 (2014).

40. Tadano, T., Gohda, Y. \& Tsuneyuki, S. Impact of rattlers on thermal conductivity of a thermoelectric clathrate: a first-principles study. Phys. Rev. Lett. 114, 95501 (2015).

41. Zhang, $\mathrm{H}$. et al. Atomic interactions in the p-type clathrate $\mathrm{I} \mathrm{Ba}_{8} \mathrm{Au}_{5.3} \mathrm{Ge}_{40.7}$. Inorganic Chemistry 50, 1250-1257 (2011).

42. Johnsen, S., Christensen, M., Thomsen, B., Madsen, G. K. H. \& Iversen, B. B. Barium dynamics in noble-metal clathrates. Phys. Rev. B 82, 184303 (2010).

43. Ye, Z. et al. Thermoelectric properties of Au-containing type-I clathrates $\mathrm{Ba}_{8} \mathrm{Au}_{\mathrm{x}} \mathrm{Ga}_{16-3 \mathrm{x}} \mathrm{Ge}_{30+2 \mathrm{x}}$. J. Alloys Compd. 587, 747-754 (2014).

44. Ormeci A. \& Grin Yu. Coexistence of ionic and covalent atomic interactions (bonding inhomogeneity) and thermoelectric properties of intermetallic clathrates. J. Thermoelectr. 6, 16-32 (2016).

45. Kulda, J., Debernardi, A., Cardona, M., De Geuser, F. \& Haller, E. E. Self-energy of zone-boundary phonons in germanium: $\mathrm{Ab}$ initio calculations versus neutron spin-echo measurements. Phys. Rev. B 69, 45209 (2004).

46. Larkin, J. M. \& McGaughey, A. J. H. Thermal conductivity accumulation in amorphous silica and amorphous silicon. Phys. Rev. B 89, 144303 (2014).

47. Baldi, G. et al. Thermal conductivity and terahertz vibrational dynamics of vitreous silica. Phys. Rev. B 77, 214309 (2008).

48. Baldi, G., Giordano, V. M., Monaco, G. \& Ruta, B. Sound attenuation at terahertz frequencies and the boson peak of vitreous silica. Phys. Rev. Lett. 104, 195501 (2010)

49. Slack, G. A. Thermal conductivity of elements with complex lattices: B, P, S. Phys. Rev. 139, A507 (1965).

50. Roufosse, M. \& Klemens, P. G. Thermal conductivity of complex dielectric crystals. Phys. Rev. B 7, 5379 (1973)

51. Slack, G. A. The thermal conductivity of nonmetallic crystals. Solid state Phys. 34, 1-71 (1979)

52. Morelli, D. T. \& Slack, G. A. in High Therm. Conduct. Mater. 37-68 (Springer, 2006).

53. Takeuchi, T., Nagasako, N., Asahi, R. \& Mizutani, U. Extremely small thermal conductivity of the Al-based Mackay-type 1/1-cubic approximants. Phys. Rev. B 74, 54206 (2006).

54. de Boissieu, M. et al. Lattice dynamics of the $\mathrm{Zn}-\mathrm{Mg}-\mathrm{Sc}$ icosahedral quasicrystal and its Zn--Sc periodic 1/1 approximant. Nat. Mater. 6, 977-984 (2007).

55. de Boissieu, M., Currat, R., Francoual, S. \& Kats, E. Sound-mode broadening in quasicrystals: A simple phenomenological model. Phys. Rev. B 69, 54205 (2004).

56. Kaviany, M. Heat Transfer Physics (Cambridge University Press, 2014).
57. Liao, B. et al. Significant reduction of lattice thermal conductivity by the electron-phonon interaction in silicon with high carrier concentrations: a firstprinciples study. Phys. Rev. Lett. 114, 115901 (2015).

58. Sales, B. C., Chakoumakos, B. C., Jin, R., Thompson, J. R. \& Mandrus, D. Structural, magnetic, thermal, and transport properties of $\mathrm{X}_{8} \mathrm{Ga}_{16} \mathrm{Ge}_{30}(\mathrm{X}=\mathrm{Eu}$, Sr, Ba) single crystals. Phys. Rev. B 63, 245113 (2001).

59. English, N. J., John, S. T. \& Carey, D. J. Mechanisms for thermal conduction in various polymorphs of methane hydrate. Phys. Rev. B 80, 134306 (2009).

60. Liu, Y., Xi, Q., Zhou, J., Nakayama, T. \& Li, B. Phonon-glass dynamics in thermoelectric clathrates. Phys. Rev. B 93, 214305 (2016).

61. Chakraborty, A. \& Bouzerar, G. Long wavelength spin dynamics in diluted magnetic systems: Scaling of magnon lifetime. J. Magn. Magn. Mater. 381, 50-55 (2015).

62. Hellman, O. \& Broido, D. A. Erratum: Phonon thermal transport in $\mathrm{Bi}_{2} \mathrm{Te}_{3}$ from first principles. Phys. Rev. B 92, 219903 (2015).

63. Cahill, D. G. Thermal conductivity measurement from 30 to $750 \mathrm{~K}$ : the $3 \omega$ method. Rev. Sci. Instrum. 61, 802-808 (1990).

64. Ikeda, M. et al. Multiband transport in $\mathrm{CoSb}_{3}$ Prepared by Rapid Solidification,. Zeitschrift für Anorg. und Allg Chemie 641, 2020-2028 (2015).

65. Ramlau, R. \& Grin Yu, S. H. Atomic resolution microscopy of intermetallic clathrates. JEOL News 51, 2-6 (2016).

\section{Acknowledgements}

This work was carried out within the European C-MAC network. We thank the ILL and the LLB for the attribution of beam time. M.M. has been supported by Slovak science agency project APVV-15-0621 and CNRS. VASP calculations were performed in the Computing Centre of the Slovak Academy of Sciences using the supercomputing infrastructure acquired in project ITMS 26230120002 and 26210120002, funded by ERDF. The work of M.d.B. was supported by the ANR 2011-BS04-004-01. S. Pailhès acknowledges support from the French National Research Agency (ANR-13-PRGE0004) and S. Paschen from the Austrian Science Fund (FWF TRP-176-N22). Y.G. and M.B. acknowledge the support of the DFG SPP 1415.

\section{Author contributions}

Single grain growth and characterization, together with structure determination by X-ray diffraction and TEM analysis were carried out by Y.G., H.D.N., R.R., H.B., M.S., M.B., C.A. Neutron experiments and analysis were carried out by P.-F.L., S. Pailhès, V.M.G., M.d.B., with F.B. and L.P.R. (IN22-ZETA, ILL), J.O. (IN5, ILL), Y.S. (2T, LLB). The measurements of the thermal conductivity were performed by S. Paschen, M.I. and P.T. Ab initio DFT simulations was carried out by P.-F.L., H.E., M.R.J. and M.M. The phenomenological model was developed by S. Pailhès and further discussed by M.d.B. and V.M.G. Data and simulation interpretation has been carried out by P-F.L., S. Pailhès, V.G., M.d.B., Y.G., M.B., S. Paschen, M.I., P.T., H.E., H.S., and M.R.J. S. Pailhès, V.M.G., P.-F.L. and M.d.B. co-wrote the paper. All authors commented and contributed to the final version of the manuscript. The neutron investigations and ab initio simulations were performed in the context of the $\mathrm{PhD}$ work of P.-F.L. which has been financially supported by the ILL and co-supervised by H.S., M.R.J., S. Pailhès, V.M.G., and M.d.B.

\section{Additional information}

Supplementary Information accompanies this paper at doi:10.1038/s41467-017-00584-7.

Competing interests: The authors declare no competing financial interests.

Reprints and permission information is available online at http://npg.nature.com/ reprintsandpermissions/

Publisher's note: Springer Nature remains neutral with regard to jurisdictional claims in published maps and institutional affiliations.

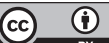

Open Access This article is licensed under a Creative Commons Attribution 4.0 International License, which permits use, sharing, adaptation, distribution and reproduction in any medium or format, as long as you give appropriate credit to the original author(s) and the source, provide a link to the Creative Commons license, and indicate if changes were made. The images or other third party material in this article e-Proof the figure resolution will appear at lower resolution than in the pdf and html versions of your paper.)"-->are included in the article's Creative Commons license, unless indicated otherwise in a credit line to the material. If material is not included in the article's Creative Commons license and your intended use is not permitted by statutory regulation or exceeds the permitted use, you will need to obtain permission directly from the copyright holder. To view a copy of this license, visit http:// creativecommons.org/licenses/by/4.0/.

(C) The Author(s) 2017 